\title{
L'ESPRIT NOVATEUR CHEZ APOLLINAIRE: L' UN DES AVENTURIERS DE LA POESIE MODERNE
}

\author{
"A LA FIN TU ES LAS DE CE MONDE ANCIEN" \\ Alcools. (Zone), p:7
}

\author{
Dr: Attia El-Imam Al-kolali \\ Maître de conférences- Faculté des lettres. Damiette- Université \\ de Mansoura
}


Dès le début du XXème siècle, l'emblème «changer la vie», lancé par les surréalistes, envahit la vie littéraire et artistique de la Belle Epoque et des Années Folles. Romanciers et poètes s'affranchissent des canons traditionnels pour côtoyer le désir créatif. Ainsi, de nouvelles orientations se dessinent et s'enracinent plus nettement, et une nouvelle génération de poètes est affectée par les technologies nouvelles avec un rythme accéléré.

En fait, le début de ce siècle est distingué par une nuance avec les héritages du XIXème siècle, qu'il s'agisse de la perpétuité du mouvement symboliste et décadentiste avec Prudhomme, Anna de Noailles, ou qu'il s'agisse des voyageurs comme Blaise Cendrars [La prose du Transsibérien, 1913], Guillaume Apollinaire [Alcools, 1913, Calligrammes, 1918], Victor Segalen [Stellès, 1912] qui parcourent «l'Esprit nouveau» en s'occupant à la fois des thèmes et des formes du texte poétique par le refus de toute séparation entre art et vie quotidienne, par la priorité donnée à l'expression de l'instantané et du simultané, par la recherche méthodique de la surprise. Et c'est alors qu'apparaîssent ce qu'on appelle «les poètes de l'esprit nouveau».

Apollinaire, l'un des poètes illustres de cette période, jouissait d'une science considérable pour son temps, complétée par divers voyages. Ses poèmes contiennent idées et pensées qui sont purement propres à lui: des idées philosophiques, des événements historiques et vrais. Il cherche à «saisir la vie dans sa complexité et ses contradictions, être sensible à tous les appels du monde moderne.» (1)

L'auteur d'Alcools annonce «les iconoclastes créateurs» par son pouvoir prophétique, par son souci à ne rien laisser perdre, machines, trains, avions, et la nouveauté le grise. Il fait entrer la poésie française dans le tourbillon du XXème siècle avec des poèmes - promenades, des poèmes d'un parcours long et rapide, des voyages et des rêves. Et tout se traduit par des couleurs, des images, des gestes, des mouvements accompagnés d'un jeu de l'ombre et de la lumière propres aux peintres.

Avec une vitesse vertigineuse, impressions, souvenirs et imaginations s'enchevêtrent et se superposent selon un rythme accéléré, presque automatique et improvisé. Et tout lecteur est invité à parcourir les océans, les déserts, les routes «sous le Pont 
Mirabeau», dans «le Transsibérien». C'est l'aventure, c'est le rythme du monde moderne.

Apollinaire mit en relief les thèmes modernes [la ville, le voyage, la vitesse, le feu .... ], en employant les images qui laissent un impact sur le coeur et l'âme du lecteur, et enfin l'écriture moderne, libérée et témoin essentiel de la poésie du XXème siècle [disparition de la rime, de la ponctuation, des vers métrés], et en choisissant les mots, les sons et les pensées ayant leur musique et leur rythme.

Dès le premier vers d' «싣ools», Apollinaire, en s'adressant à la Tour Eiffel, annonce sa lassitude de «ce monde ancien» et son goût pour la modernité. Sa soif pour la modernité dépasse même les choses les plus modernes. Il est touché par le progrès au rythme extrêmement rapide en citant ses aspects comme: «les journaux, les livraisons à 25 centimes pleines d'aventures policières, les directeurs, les ouvriers, les belles sténo - dactylographes et les troupeaux d'autobus.»

Ces aspects peuvent assouvir l'ambition du poète à un idéal: «J'aime la grâce de cette rue industrielle». Mais l'invention qui égare les esprits est sans doute l'avion, qui rend l'errance moins douloureuse: de Marseille à Coblence, de Rome à Amsterdam, .... Le monde devient un petit village facile à parcourir. Les êtres mystérieux, prophètes, prêtres sont tous fascinés par le génie de cette découverte:

«Les diables dans les abîmes lèvent la tête pour regarder Ils disent qu' il imite Simon Mage en Judeé ....

Les anges voltigent autour du joli voltigeur» Alcools, (Zone), p:9.

Apollinaire anime l'inanimé [la Sirène aboie, les autobus mugissent, «L'avion se pose enfin sans refermer ses ailes» attirant dans son sillage des millions d'oiseaux quotidiens, exotiques, fabuleux venus fraterniser «avec la volante machine»]. Ce choix est évidemment révélateur:

«Apollinaire s' approprie le monde, il ne le perd ni le subit. Les choses rentrent dans I' ordre humain, les hommes ne peuvent être réifiés.» (2)

Il unit les objets et les hommes dans un même univers poétique où les choses, comme douées de pouvoirs magiques, peuvent parfois influer sur certains hommes:

«La table et les deux verres devinrent un mourant qui 
nous jeta le dernier regard d'Orphée

Les verres tombèrent se brisèrent

Et nous apprîmes à rire" Alcools, (poème lu au mariage d'ASalomon), P:59.

Est- ce une scène morbide ou une «Comédie humaine»?

Dans ce quatrain, le rapprochement et la suggestion

s'élargissent progressivement pour admettre toujours plus de réalité quotidienne, et en plus la modernité d'Apollinaire réside dans sa quête, dans sa remise en question de soi- même et du monde, qui demeure évidemment encore actuelle.

Chez Apollinaire, l'écoulement des choses mérite une attention spéciale, c'est l'eau du fleuve, version concrète de la métaphore usée $d$ ' «Automne malade»: «La vie s'écoule» Alcools, (Automne malade), p:132.

L'eau se présente comme un élément féminin: féminine, dans la majorité des poèmes d' «Alcools», par ses créatures mythologiques, les «déités» qui «laissent couler leurs cheveux» (Clotilde) p:47, par le rappel de la femme aimée, l'ordre et le véhicule mélancolique destiné à emporter, sous «Le Pont Mirabeau», les jours, les semaines et les amours du poète.

Chez Apollinaire, ce n'est plus le temps romantique ni le temps classique: C'est l'écoulement du temps qui se confond avec les images de l'eau, symbole de l'instabilité des choses.

Le passage de l'eau est régulièrement associé à l'agonie automnale dont il représente la voix murmurante ou grave.

Le contexte expressif de l'eau, soit de la rivière ou de la mer, est souvent imbibé de tristesse funèbre. Telle la «profonde eau triste» où vont les «pi- mus couples», sur un fond sonore de mort:

«J'entends mourir et remourir un chant lointain» Alcools, (La porte), $\mathrm{p}: 64$.

Par son caractère fuyant et insaisissable, l'eau s'intègre au processus de la mort car elle cause la destruction.

Apollinaire avait d'abord songé à intituler son recueil «Eau- devie», cependant, «Alcools» est plus net, provocant et moderne, exprimant l'acte poétique, dans sa continuité avec Baudelaire et Rimbaud.

«Ecoutez mes chants d'universelle ivrognerie» Alcools, (Vendémiaire), $\mathrm{p}: 142$.

En fait, les références explicites à la boisson énivrante sont nombreuses. Apollinaire buvait- il beaucoup dans sa vie 
personnelle?

«Et tu bois cet alcool brûlant comme ta vie

Ta vie que tu bois comme une eau- de- vie» Alcools (Zone), $\mathrm{p}: 14$.

«Nous fumons et buvons comme autrefois» Alcools, (poème lu au mariage d'André Salomon), p:60.

"Mon verre est plein d'un vin trembleur comme une flamme" Alcools, (Nuit Rhénane), p:94.

De même, l'univers d'«Alcools» est couronné de nombreux lieux fournisseurs de boissons: des «tavernes» (Zone), des auberges, celle du «Voyageur» est «triste» et celles des «Saltimbanques» sont «grises», et des brasseries:

«Beaucoup entraient dans les brasseries» Alcools, (La maison des morts), p:45.

«Elle ... buvait lasse des trottoirs

Très tard dans les brasseries borgnes» Alcools, (Marizibill), p:51.

Le symbolisme du titre, sa multifonction s'élargit, l'alcool désigne l'universelle soif du poète, l'apogée de ses désirs:

«Je buvais à pleins verres les étoiles» Alcools, (Les

fiançailles), p:115.

«Je suis ivre d'avoir bu tout l'univers ...

Et je boirai encore s'il me plaît l'univers» Alcools, (Vendémiaire), $\mathrm{p}: 142$.

Extrême et intarissable, cette soif, souvent euphorique, court le risque de demeurer inassouvie:

«Mondes ... Je vous ai bus et ne fus pas désaltéré» Alcools, (Vendémiaire), p:142.

L'alcool suggère, en outre, le dépassement, la possibilité de refuser les traditions et les normes, et d'encourager une poésie novatrice et moderne.

Dans «Poème lu au mariage d'André Salomon», l'eau de fleuve ne court plus, puisqu' il permet la présence de "nénuphars", plantes des eaux stagnantes:

«Le fleuve sur lequel flottait Ophélie.

Qui blanche flotte encore entre les nénuphars» Alcools, p:59.

Eaux dormantes dont la quasi- immobilité évoque celle des fleuves infernaux, le Styx et le Léthé, elles prolongent l'état de mort, comme l'eau du «lac blanc» de (La chanson du malaimé):

«Un jour le roi dans l'eau d'argent

se noya puis la bouche ouverte 
Il s'en revint en surnageant

Sur la rive dormir inerte

Face tournée au ciel changeant» Alcools, p:31.

Les images d'écoulement, de longueur, comme les boyaux, les cordes et les tours, suggèrent un mouvement continuel, parfois circulaire. Certaines images sont dynamiques dans le sens baudelairien, la présence intangible et changeante de fumées, de flammes, de parfums, et d'aube. L'image des fleurs est parfois associée à celle du liquide:

«La fusée s'épanouit fleur nocturne Quand il fait noir

Et elle retombe comme une pluie de larmes amoureuses»

Ces images de fleurs qui éclosent et de liquides qui s'écoulent suggèrent le mouvement et l'éphémère, mais un moindre degré que celles de l'éclatement d'un obus.

En fait, les images liquides embellissent «Calligrammes»: la pluie, les fleuves, le monde est liquide et les êtres humains le sont aussi: «Maison humide ... bouteilles champenoises où le sang fermente» (Océan de terre), p:134.

Le flux de l'eau est fréquemment, d'une façon renouvelée, associé au temps qui passe, à la fois irréversible «passent les jours et passent les semaines / Ni temps passé / Ni les amours reviennent / Sous le Pont Mirabeau coule la seine» ( Le Pont Mirabeau ) p:16, et immuable «Je passais au bord de la seine / Un livre ancien sous le bras / Le fleuve est pareil à ma peine / Il s'écoule et ne tarit pas / Quand donc finira la semaine» Alcools, (Marie), p:56.

L'alcool peut évoquer la soif, le désir de consommer la vie, la soif est synonyme de curiosité, d'enthousiasme, de désir intense. Il est caractérisé par le tremblement qui accompagne l'union des contraires:

«Mon verre est plein d'un vin trembleur comme une flamme Le Rhin le Rhin est ivre où les vignes se mirent

Tout l'or des nuits tombe en tremblant s'y refléter» Alcools, (Nuit Rhénane), p:94.

Comme un peintre, Apollinaire utilise le rouge et l'or pour dessiner une image puissante qui montre sa vision de ce nouveau monde. Le rouge représente la passion et symbolise les émotions fortes. L'or symbolise l'éternel et contient un aspect spirituel.

Le poète mélange les aspects contradictoires: le provisoire et l'éternel se mêlent ici pour créer une image du monde, où les 
valeurs antiques affrontent les nouvelles idées du XXème siècle.

Dans «Nuit Rhénane», le vin s'oppose à l'eau, à la fatalité automnale, symbolisée par le batelier et les sept filles des eaux. Le poème s'achève par la victoire de l'ivresse sur la voix de mort par un geste euphorique du narrateur:

«Mon verre s'est brisé comme un éclat de rire» Alcools, p:94.

Ce poème est chronologiquement le premier où apparaîsse l'alcool, qui est en même temps eau et feu: «L'eau de vie, dit Bachelard, c'est l'eau de feu». (3)

Dans ce poème, le verbe "trembler", évoqué par le mélange de la nuit de l'eau, de la vie et de la mort, apparaît à deux reprises à l'occasion de l'union d'un liquide et d'une flamme. La puissance du vin est grande: le fleuve lui même semble saisi par l'ivresse. En plus, l'alcool apparaît comme un remède à la fatalité. Sur le plan cosmologique et mythologique, l'eau, devenue alcool, change de signe et s'humanise : $C$ e ne sont pas les êtres qui sont ivres, mais le Rhin: «Le Rhin le Rhin est ivre ... » Alcools, (Nuit Rhénane), $\mathrm{p}: 94$.

C'est dans «Vendémiaire», poème dionysiaque, que se développe la figure de l'alcool. Le poème a pour objet une immense vendange miraculeuse, poème métaphorique où les rivières remontent leur cours pour atteindre la gorge du poète, l'automne change de signe pour devenir la saison de la vendange poétique. Le monde, métamorphosé en alcool, est absorbé par le poète, qui fait couler dans la gorge de Paris les villes de France, d'Europe et du monde entier «Le Rhône, la Saône, la Méditerranée, la Moselle, Lyon, le Rhin, la Grèce ancienne, l'Orient, la Bretagne légendaire, Ixion et le Vatican».

Apollinaire, plus avide que la cité, est un poète que les villes, changées en vin pur, ne suffisent pas à désaltérer:

«Je vous ai bus et ne fus pas désaltéré

Mais je connus dès lors quelle saveur a l'univers

Je suis ivre d'avoir bu tout l'univers ...

Ecoutez- moi je suis le gosier de Paris

Et je boirai encore s'il me plaît l'univers» Alcools, (Vendémiaire), $\mathrm{p}: 142$.

L'univers métamorphosé en paroles contenant son essence et son sang, s'offre à Paris car il est la métropole, la Rome de la poésie, et chacun des poèmes est un alcool, c'est- à- dire une métamorphose du monde en chant, sa résurrection permet à l'homme de s'unir à l'univers au lieu d'être à sa merci.

L'alcool apollinairien n'est plus le baudelairien«vin de 
solitaire». Il présente une espèce $d$ ' ivresse euphorique et cosmique, une inflammation lyrique, la saoulerie «des chants d'universelle ivrognerie».

Chez Apollinaire, l'alcool établit un équilibre entre la vie et la poésie qui, en lui, se mélangent en une même intensité ou brûlure. La fin de «Zone» l' exprime nettement par l'image d'absorption d'alcool, assimilé à la vie même:

«Et tu bois alcool brûlant comme ta vie

Ta vie que tu bois comme une eau- de- vie» Alcools, (Zone), p:14.

L'eau, cet élément relevé par Bachelard, se trouve côte à côte avec le feu chez Apollinaire. Ce feu est doué d'une double postulation et porte en lui- même une nouvelle "muse", trait d'innovation chez le poète: feu du bûcher qui purifie et délire, feu solaire qui symbolise les hauteurs sidérales de la flamme poétique. Dans «La chanson du mal- aimé», cette double nature est sensible et sensibilisée:

«Juin ton soleil ardente lyre

Brûle mes doigts endoloris

Triste et mélodieux délire» Alcools, p:31.

Le mouvement d'exaltation par lequel débute «Le brasier» évoque l'atmosphère où se situe le sacrifice par le feu; celui de l'enthousiasme divin: «J'ai jeté dans le noble feu

Que je transporte et que j'adore

De vives mains et même feu

Ce passé ces têtes de morts

Flamme je fais ce que tu veux» Alcools, (Le brasier), p:89.

Dans cette strophe, le poète se présente comme le lieu de l'holocauste: il rêve du feu qui brûle son passé, et il devient luimême à la fois le lieu et le comestible du feu:

«Je flamme dans le brasier à l'ardeur adorable

Et les mains des croyants m'y rejettent multiple innombrablement

Je suffis pour l'éternité à entretenir le feu de mes délices» Alcools, (Le brasier), p:91.

Le poète veut se purifier, se débarrasser de son passé et de son amour, la purification se fait dans la douleur, et il avoue à la fin des «Fiançailles» que le bûcher est «le nid de son courage» qui crée un univers nouveau, une «vie renouvelée». De plus, tel un Lamartine ou un Hugo, il avait le sentiment raciste d'appartenir à un rang supérieur des poètes pionniers: 
«ll n'y a plus rien de commun entre moi

Et ceux qui craignent les brûleurs» Alcools, (Le brasier), p:91.

En fait, l'image du bûcher montre une nouvelle figure du poète, acquise par l'épreuve du feu:

«Templiers flamboyants je brûle parmi vous prophétisons ensemble ô grand maître je suis le désirable feu qui pour vous se dévoue ... Liens déliés par une libre flamme Ardeur Que mon souffle éteindra ô Morts à quarantaine» Alcools, (Fiançailles), p:122.

Les «Templiers» dont il s'agit furent brûlés en 1314, en période de carême "quarantaine", et l'un d'eux annonça par avance la mort de Clément V et de Philippe le Bel. (4)

Concernant l'aspect solaire du feu, le poète a pris son vol dans les hauteurs sidérales et il a participé alors à la nature solaire: «... ma sagesse égale

Celle des constellations

Car c'est moi seul nuit qui t'étoile » Alcools, (Lul de Faltenin), $\mathrm{p}: 77$.

Les images modernes qui assimilent le poète à un corps astral, flamboyant, comète, étoile, soleil, se trouvent dans plusieurs poèmes. Une des plus intéressantes, dans «Le brasier» est celle qui touche la situation du poète à une altitude intersidérale: « Au-delà de notre atmosphère s'élève un théâtre Que construisit le ver Zamir sans instrument Là- haut le théâtre est bâti avec le fer solide Comme les astres dont se nourrit le vide» Alcools, (Le brasier), p:92.

Le ver Zamir, pour Apollinaire, est «l'animal fabuleux symbolisant tout à coup la puissance créatrice, l'architecte de l'imaginaire.» (5)

Ainsi le poète bâtit- $t$ - il son œuvre «sans instrument», avec le pouvoir magique des mots «changés en étoiles». Quel renouvellement du verbe poétique! Ce changement de la vision créatrice et l'idée de l'élévation solaire ont fait du poète un frère d'lcare, l'oiseau- poète de «Cortège»: «Oiseau tranquille au vol inverse oiseau Qui nidifie en l'air» Alcools, (Cortège), p:48.

Ailleurs, la métaphore, dont l'allitération renforce l'efficacité, change le spectacle initial d'un coucher de soleil en une scène de décapitation: «Soleil cou coupé» (Zone), p:14. 
Le feu, second élément, se retrouve chez son contemporain, Blaise Cendrars. C'est le cœur brûlant qui pousse le poète dès son arrivée en Russie au temps de la guerre russojaponaise: «Mon cœur brûlait.»

Le feu devient le combustible cher pour sa création poétique: «L'écriture est un incendie qui embrase un grand remueménage d'idées et qui fait flamboyer des associations d'images avant de les réduire en braises crépitantes et en cendres retombantes. Mais si la flamme déclenche l'alerte, la spontanéité du feu reste mystérieuse. Car écrire c'est brûler vif, mais c'est aussi renaître de ses cendres.» (6)

Gaston Bachelard n'a t- il pas à plusieurs reprises soulevé la question du feu?

«Dis- moi quel est ton infini, je saurai le sens de ton univers, est- ce l'infini de la mer ou du ciel, est- ce l'infini de la terre profonde ou celui du bûcher?» (7)

C'est le feu qui mène Blaise Cendrars à voyager ambulant, cet éternel errant du cosmos: «Je suis en route ». Cette fois- ci le moyen de transport n'est pas le bateau de Baudelaire mais le Transsibérien qui symbolise la modernité d'alors. Le poète retient les cadences du train en folie, alternant avec la parole de la petite Jehanne:

« Dis, Blaise, sommes- nous bien loin de Montmartre?» (La Prose du Transsibérien)

Dès les premiers vers de «La prose du Transsibérien", le rouge du feu et du sang donne la couleur du poème: Celle d'une course infernale dans laquelle «tous les démons sont déchaînés» et «tous les trains sont les bilboquets du diable», qui prend l'allure d'une plongée dans l'abîme à mesure que l'on approche de la fin:

«La mort en Mandchourie

Est notre débarcadère est notre dernier repaire» (La Prose du Transsibérien)

Le transsibérien est accompagné des maladies, des famines: «la faim, la putain, se cramponnent aux nuages en débandade et fiente des batailles en tas puants de morts», de la guerre, des souffrances: «J'ai vu dans les lazarets les plaies béantes les blessures qui saignaient à pleines orgues», allusions au temps de la première guerre mondiale, et le voyage laisse le poète en proie à la nostalgie de sa «jeunesse perdue» et au regret: «Je voudrais n'avoir jamais fait mes voyages».

Chez Cendrars, les évocations de la guerre et des visions 
d'horreur sont les motifs qui rythment à la fois le poème et le voyage: «La peste le choléra se lèvent comme des braises ardentes sur notre route / Nous disparaissons dans la guerre en plein dans un tunnel». (La Prose du Transsibérien)

En fait, la machine occupe une place de plus en plus grande dans la poésie du XXème siècle surtout chez Apollinaire et Cendrars annonçant l'avènement de l'homme planétaire, et exaltant les machines et la technique. Leurs poèmes ont- ils influencé l'essor industriel de la France des années 1920?

Dans «Les Fiançailles», la belle femme devient une machine, le fer est son sang, la flamme son cerveau. Apollinaire montre son enthousiasme pour la révolution industrielle et scientifique: «J'aimais j'aimais le peuple habile des machines» Alcools, (Les Fiançailles), p:125.

Le luxe et la beauté sont des produits de cette révolution industrielle, et l'idéal féminin devient un symbole de progrès. Cette dame à la beauté frappante représente la France, lieu du progrès:

«Les yeux bleus les dents blanches et les lèvres très rouges Elle avait un visage aux couleurs de France» Alcools, (Les Fiançailles), p:124.

Certes, le progrès technique et scientifique envahit plusieurs domaines: l'automobile, l'aviation, les télécommunications, l'électricité, etc ...

Ensuite, les rapports entre les hommes se modifient grâce aux progrès techniques appliqués aux transports et à la transmission du son et des images. Le train roule dans «le soir d'été», les rails traversent «la chanson du mal- aimé» et les tramways «feux verts sur l'échine»y «musiquent», l'omnibus «grince» dans «la bonne chanson» parmi les lueurs rouges et vertes:

«L'omnibus, ouragan de ferraille et de boues

Qui grince, mal assis entre ses quatre roues

Et roule ses yeux verts et rouges lentement»

Métaux et métallurgie, fer et ferrailles semblent dorénavant intéresser Apollinaire, fasciné par les usines qui sont plus hautes que les églises:

«Le style moderne existe, mais ce qui caractérise le style d'aujourd'hui, on le remarquerait moins dans les façades des maisons ou dans les meubles que dans les constructions de 
fer, les machines, les automobiles, les bicyclettes, les aéroplanes ... Le chef-d'œuvre de style moderne est en fonte, en acier, en tôle» (8) déclare- $t$ - il.

Dès le début de «Zone», Apollinaire penche vers tout changement, vers la modernité: «automobiles» et «avions», mode de transport rapide facilitant la vie quotidienne, évoquant la «rue industrielle» fréquentée par les employés:

«Du lundi matin au samedi soir quatre fois par jour y passent» Alcools, (Zone), p:8.

L'intégration de la machine à la poésie mène les critiques à considérer Apollinaire parmi les poètes futuristes: «la poésie s'emparera des grands domaines neufs proposés à l'homme par l'activité des machines et des découvertes scientifiques», dit- il dans une conférence sur «L'Esprit nouveau et les poètes» en 1917.

Dans «Zone», Apollinaire aborde le domaine de l'aviation à travers le «Christ»:

«C'est le Christ qui monte au ciel mieux que les aviateurs Il détient le record du monde pour la hauteur» Alcools, (Zone), $\mathrm{p}: 9$ et également à travers les anges:

«Les anges voltigent autour du joli voltigeur

Flottent autour du premier aéroplane» Alcools, (zone), p:9.

C'est presque un panorama artistique où se confond le prophète et le «Christ», «les anges», «l'avion» et les «oiseaux»:

«L'avion se pose enfin sans refermer les ailes

Le ciel s'emplit alors de millions d'hirondelles

A tire-d'aile viennent les corbeaux les faucons les hiboux» Alcools, (Zone), p:9.

Apollinaire transforme le «Christ» en aviateur et décrit la ville industrielle moderne tout en chantant les machines de guerre, les télégraphes, les canons, les obus, etc ...

Mais son modernisme n'oublie pas le monde ancien, le monde de l'«Olympe» et de la «Bible».

Apollinaire est considéré comme un lyrique moderne, qui mêle lyrisme et modernisme dans sa poésie. Il est satisfait que le XXème siècle ait opéré un changement de l'homme, dans son expression, dans sa sensibilité et dans ses liens avec l'Autre. Les nouveaux moyens de communication et d'expression tendent à la technologie et la poésie se doit réagir face à ce monde renouvelé. C'est pourquoi il préfère l'intégration du monde nouveau dans la poésie. Cette idée réactualise celle de Baudelaire. 
Dans «Zone», Apollinaire crée un conflit entre le monde industriel et la nature en choisissant une terminologie propre à la nature:

«Des troupeaux d'autobus mugissants près de toi roulent» Alcools, (Zone), p:10.

Ce vers montre le mélange des aspects naturels et industriels. Ici, l'autobus est transformé en un animal par les mots «mugissants» et «troupeaux», un animal sauvage à cause du bruit qu'il fait. La dureté du son «r» dans le mot «roulent» montre la dissymétrie de cette atmosphère. Et «près de toi» exprime le contraste entre les autobus, aspect moderne, fabriqués de métal et le «toi» présente une personne faite de chair. Cet aspect cubiste consiste à mêler les inventions modernes avec les aspects de la nature primitive.

Roberto Tassari a déclaré que «le futurisme de Marinetti ne tendait qu'à briser idéalement la liaison entre machine et système économique, pour enseigner aux travailleurs non la leçon qui vient de l'économie, mais celle qu'on peut tirer du mythe de la Machine abstraite, devenue objet d'amour tristanique.» (9)

Chez Apollinaire, les lieux sont variés: lors d'un voyage, «Le voyageur» et «Hôtels» en témoignent. Il s'agit de «gens du voyage» dans les «Saltimbanques», «La tzigane» et dans d'autres poèmes:

«Un ours un singe un chien menés par les tziganes» Alcools (Mai), p:95.

«Des sorciers venus de Bohême» Alcools, (Crépuscule), p:37.

Le voyage est fréquemment rapporté à l'expérience personnelle du poète dans un espace ouvert:

«Maintenant tu es au bord de la Méditerranée ... Avec tes amis tu te promènes en barque ...

Te voici à Amsterdam avec une jeune fille ...» Alcools, (Zone), p:11.

Le voyage sur le Rhin est un thème romantique, traité par Victor Hugo par des dessins intéressants à travers des encres.

Apollinaire a ajouté des poèmes inspirés par sa découverte des bords du Rhin, avec leurs auberges, leur vin blanc, leurs ruines, leurs mythes, leurs filles. N'a- $t$ - il pas passé quelques années comme précepteur?

Dans «Nuit Rhénane», la nuit est l'espace du rêve et des légendes. La nuit rhénane est aussi la nuit de l'ivresse dans le mouvement des aspects concurrents de la réalité et de la fable. 
Ce poème est un voyage au bord du Rhin dans lequel le poète mêle réel et irréel, Apollinaire y montre le modernisme avec l'absence de la ponctuation, s'inspirant beaucoup de la mythologie; il voyage en Allemagne dont le fleuve du Rhin représente un obstacle, mais aussi une source d'inspiration, une source de vie où il se noie.

Le passé du poète est représenté par le biais des mythes, nombreux dans le recueil et très divers: la Bible, les contes populaires, les légendes gréco- latines, orientales, Celtiques, germaniques, etc ... et ils contribuent, par leur exotisme et leur étrangeté, au charme mystérieux et nostalgique qui enrichit et rapproche de nous le monde moderne.

D'ailleurs, le voyage, spatial ou temporel, est un signe de la liberté et peut donc être associé à la fête et à la richesse: les Saltimbanques «ont des poids ronds ou carrés (Saltimbanques), p:68.

Des tambours des cerceaux dorés" Alcools,

Apollinaire signale la toute- puissance de l'imagination poétique:

«Vers le palais de Rosemonde au fond du rêve Mes rêveuses pensées pieds nus vont en soirée ... Pour mes pensées de tous pays de tous les temps.» Alcools, (Palais), p:34.

Cet aspect positif de voyage, qui supprime les limites et les entraves, a son envers négatif: dépourvu de but précis, le voyage est avant tout une errance, un symbole d'une douloureuse méconnaissance de soi:

«Temps passés Trépassés les dieux qui ne formâtes Je ne vis que passant ainsi que vous passâtes

Et détournant mes yeux de ce vide avenir

En moi- même je vois tout le passé grandir» Alcools, (Cortège), p:50.

Au poème «Voyage» rédigé par Apollinaire, correspond le poème de «La prose du Transsibérien» de Blaise Cendrars présentant une aventure ferroviaire. Ce poème semble prouver qu'il existe une «poésie du rail». Blaise Cendrars, comme les peintres et les poètes de toutes les avant-gardes, s'enchante de la machine; de la vitesse, de l'avion ... où l'imaginaire se mêle au réel d'une façon inextricable:

«Le train fait un saut périlleux et retombe sur toutes ses roues Le train retombe toujours sur toutes ses roues Et je construirai un hangar pour mon avion avec les os 
fossiles» (La Prose du Transsibérien)

Ce long poème, rythmé par le bruit et le mouvement de train, réalise l'alliance entre la poésie et la vie moderne:

«J'étais à Moscou dans la ville des milles et trois cloches et des sept gares» (La Prose du Transsibérien)

Pour Cendrars, l'entreprise du voyage apparaît comme la recherche d'un dépaysement et comme un départ de la routine du monde connu. Cela se traduit par des évocations de la distance et du mouvement : «J'ai fait courir tous les trains derrière moi», «Je suis en route», «Paris a disparu», «Nous sommes loin», «Dans les déchirures du ciel, les locomotives en furie s'enfuirent». (La Prose du Transsibérien)

Que de villes visitées! Que de monuments admirés! C'est l'époque de la construction. C'est le Beton armé et les Tours.

Apollinaire est touché par l'évolution industrielle qui évoque à son tour une évolution scientifique. II montre sa fascination par la ville, surtout par Paris dont il est épris par les rues neuves et propres, par «les directeurs les ouvriers et les belles sténodactylographes», par ses soirs lumineux et par les tramways et les feux verts.

Paris devient alors le centre de l'univers: toutes les villes de France, d'Europe et du monde entier répondent à son appel et viennent désaltérer sa soif, et la ville prend des caractères divins:

«Et Lyon répondit tandis que les anges de Fourvières Tissaient un ciel nouveau avec la soie des prières Désaltère- toi Paris avec les divines paroles» Alcools, (Vendémiaire), p:138.

Cet hommage trouve un écho à travers l'évocation des villes du nord, ces «viriles cités» qui offrent à Paris leur tribut $d^{\prime}$ '«Usines manufactures fabriques mains»:

«Et les villes du nord répondirent gaiement ô Paris nous voici boissons vivantes

Les viriles cités où dégoisent et chantent Les métalliques saints de nos saintes usines Nos cheminées à ciel ouvert engrossent les nuées Comme fit autrefois l'Ixion mécanique» Alcools, (Vendémiaire), $\mathrm{p}: 137$.

Apollinaire observe bien les innovations techniques et 
artistiques de l'époque. Pour lui, poésie et modernité sont toujours en accord et évoluent au même rythme.

On est frappé, dans «Alcools», par l'abondance des noms des lieux, surtout noms des villes. Ces signes spaciaux montrent des épisodes de la vie personnelle du poète, qui évoque sa découverte de l'Allemagne et du Rhin «La Maison des morts», «Marizibill», «Rhénanes», son séjour à Londres dans l'espoir de rattraper Annie Playden «La chanson du mal- aimé», «L'Emigrant de Lander Road», ses voyages en Hollande «Zone», «Vendémiaire».

Chez Apollinaire, la ville est un indice de la modernité, et le recueil d'«Alcools» s'ouvre et se clôt sur deux longs poèmes qui se présentent comme des parcours. «Zone» nous promène de Paris à Chartres, aux bords de la Méditerranée, à Prague, Marseille, Coblence, Rome, Amsterdam, Leyde et Gouda, avant de nous ramener à Paris, en ce quartier d'Auteuil où le poète réside alors.

A l'autre bout du recueil, «Vendémiaire» est considéré comme un véritable tour de France. Il a convoqué les capitales des provinces: Rennes, Quimper et Vannes, les villes du Nord, Lyon, les villes du midi, Rome, la Moselle et le Rhin.

Ce poème présente un panorama urbain avec une dimension internationale, comme en témoignent ces vers: «J'ai soif villes de France et d'Europe et du monde Venez toutes couler dans ma gorge profonde» Alcools, (Vendémiaire), p:136.

Dans «Zone», Apollinaire s'enthousiasme pour la Tour Eiffel, construction nouvelle et merveille entre les merveilles du monde.

Il l'a également présentée dans un calligramme pour montrer l'approche historique: Apollinaire y présente la Tour Eiffel comme un symbole de la force de la France devant les allemands:

«Salut monde dont je suis la langue éloquente que sa bouche 0 paris tire et tirera toujours aux allemands» Calligrammes, p:76.

La ville devient l'espace de la fascination, l'espace du parcours infini dont on ne se lasse pas, un espace ouvert avec ses principales composantes, ses constructions, son urbanisme. Le monde urbain parle, agit, la grande ville est présentée dans « La chanson du mal-aimé », " le Pont Mirabeau » dont le titre évoque explicitement Paris. 
L'image la plus complète et la plus apollinairienne de la ville contemporaine est dessinée dans «Zone». Le poète nous donne un tableau assez complet d'une ville industrielle dans le plein de son activité, avec ses rues grouillantes de vie, avec les sirènes des usines qui gémissent. Cette image est complètement différente de celles que nous avaient données les poètes romantiques. C'était alors la représentation de la «ville tentaculaire» qui s'opposait à l'homme, une représentation essentiellement négative qu'Apollinaire bouleverse tout à fait. Avec lui, la ville devient une âme chérie, l'abri naturel du poète et de l'homme où ils s'y plaisent grandement.

Apollinaire recourt à deux procédés en dessinant la ville contemporaine: Prolongement du mot dans le passé et vivification du mot.

Il se sert des mots nouveaux, tels que «avion, aviateur, électricité, automobile, Tour Eiffel ... ", il les plonge dans le passé, et il les met en rapport avec des mots qui sont déjà chargés de «passé», créant ainsi un lien entre les mots nouveaux et les mots qui ont une histoire et qui parfois paraissent anciens.

Pour la vivification du mot, elle apparaît à travers les images représentant les éléments de la ville moderne et les innovations de la technique. Le poète réussit à vivifier les machines par un procédé simple «soleil cou coupé», en leur donnant des voix d'animaux ou d'êtres vivants, influencé par Brague, Picasso et Delaunay:

«Bergère ô tour Eiffel le troupeau des ponts bêle ce matin» Alcools, (zone), p:7.

«Tour Eiffel» est un mot clé de ce vers; mais la semantique de ce mot, placée dans cette perspective, change complètement. Avec ce rapprochement de mots, le poète remonte dans un passé lointain: Il nous rappelle le climat et le langage des campagnards.

Dans «Zone», les exemples de vivification de mots nouveaux qui caractérisent la vie moderne sont nombreux: les prospectus, les catalogues, les affiches qui «chantent» la poésie, la sirène qui «gémit», les «inscriptions des enseignes et des murailles», les «plaques» et les «avis» qui «à la façon des perroquets criaillent», les autobus qui sont des troupeaux «mugissants».

Chez Apollinaire, les exemples des «machines vivifiées» sont clairs:

«Télégraphe oiseau qui laisse tomber ses ailes partout» Calligrammes, (Voyage), p:60. 
«Les obus miaulaient ... » Calligrammes, (Nuit d'avril), p:107. «Essaims des avions blonds ainsi que les avettes» Calligrammes, (Chant de l'horizon en Champagne), p:131. «... virilités des héros fabuleux ... pièces contre avions» Calligrammes, (Le chant d'amour), p:151. «Les avions bourdonnent ainsi que les abeilles (... ) Les réflecteurs dardant leurs lueurs comme des yeux d'escargots Et les obus en tombant sont des chiens qui jettent de la terre avec leurs pattes ... 》 Calligrammes, (A l'Italie), p:143.

Ailleurs, «la ville métallique» apparaît dans «Un soir», second aspect de la ville:

«La ville est métallique et c'est la seule étoile Noyée dans tes yeux bleus» Alcools, (Un soir), p:112.

Et dans «Le Voyageur», le spectacle de la ville n'est pas euphorique; le poète parle d'une triste auberge, de l' «orphelinat des gares»; nous trouvons aussi le «régiment des rues», la «cavalerie des ponts», le «troupeau plaintif des paysages». Cet aspect nocturne est dominé par la folie et l'ivresse. Les villes sont folles comme les machines elles- mêmes: «Les villes que $j$ 'ai vues vivaient comme des folles» Alcools, (Le Voyageur), p: 53.

Cette folie a les traits de l'ivresse, de ses titubations et de ses malaises:

«Nous traversâmes des villes qui tout le jour tournaient Et vomissaient la nuit le soleil des journées» Alcools, (Le Voyageur), p:53.

La ville apparaît comme une victime de l'ivresse «soirs de Paris ivres de gin», elle contient des lieux où l'ont peut boire d'alcool, non pas de ce vin qui réjouit le cœur de l'homme, chanté dans «Vendémiaire», mais de l'eau- de- vie qui noie les chagrins: «Tu es debout devant le zinc d'un bar Crapuleux » Alcools, (Zone), p:13.

«Les cafés gonflés de fumée » Alcools, (La chanson du malaimé), $\mathrm{p}: 32$.

Bref, l'image de la ville, pour Apollinaire, est liée à celle de Paris; quand il pense à une ville il pense à Paris avec ses monuments (Tour Eiffel, Pont Mirabeau, Sacré-Cœur), ses rues (rue Aumont- Thiéville, avenue des Ternes, rue des Rosiers, rue des Ecouffes) et ses quartiers (Montmartre, Auteuil). Il suffit de rappeler, selon une étude statistique, que le mot «Paris», dans 
Alcools, est employé vingt- deux fois, et quatorze fois dans Calligrammes. La ville donc pour lui est Paris, Paris qu'il aime chèrement:

«Paris comme une jeune fille

S'éveille langoureusement

Secoue sa longue chevelure

Et chante sa belle chanson» Calligrammes, (Les Collines), $\mathrm{p}: 28$

De son côté, Blaise Cendrars voit que la ville de Paris représente une fenêtre ouverte sur le monde offrant des visions qui forment toutes les nuances dont il a besoin pour épurer sa propre vision: «Paris ... ville de la Tour Unique»

La dame de fer constitue une source d'inspiration d'une littérature plus moderne, et l'ouvre Cendrarienne présente un cosmorama multi- dimensionnel «où le passé et le futur sont toujours dans le présent, enchevêtrés et se chevauchant». (10)

Blaise Cendrars l'a placé sous le signe de la Tour Eiffel et en quelque sorte axé sur elle:

«O Tour Eiffel/ Dieu antique/ Bête moderne/ Tour du monde/ Tour en mouvement» Du monde entier, (La Prose du Transsibérien)

La tour apparaît donc comme un point d'ancrage esthétique nouveau, universel et total.

A son tour, Emile Verhaeren veut aussi vivifier le monde moderne dans son recueil «Les villes tentaculaires» (1895), dans lequel il attaque les villes qui s'agrandissent et "vident" les campagnes.

Sa poésie est basée essentiellement sur le conflit entre la campagne qui diverge et sépare l'énergie humaine à travers ses chaumines et ses hameaux isolés, et la ville qui réunit cette énergie et la transfère en une force que l'on peut diriger contre l'adversaire.

Chez lui, les entreprises et les affaires se confondent dans une fulgurante brume:

«Automatiques et minutieux

Des ouvriers silencieux

Règlent le mouvement

D'universel titacquement

Qui fermente de fièvre et de folie.» Les villes tentaculaires, (Les Usines) 
En ce qui concerne l'écriture apollinairienne, la surprise constitue un des principes de son esthétique déclarant:

«Le nouveau existe (... ) Il est tout dans la surprise. L' esprit nouveau est également dans la surprise .... La surprise est le plus grand ressort, le plus moderne. C'est par la surprise, par la place importante qu'il fait à la surprise que l'esprit nouveau se distingue de tous les mouvements artistiques et littéraires qui l'ont précédé.» (11)

Dans «Cortège», la présence d'oiseaux légendaires ou symboliques au milieu d'oiseaux réels surprend, de même que la présence du mot grossier dans un poème lyrique. Dans le premier cas, la juxtaposition de référents appartient à des ensembles différents; dans le second, la juxtaposition de termes provient de registres différents de la langue.

Ces deux procédés sont générateurs de la surprise et de la nouveauté de l'écriture apollinairienne. «Cortège» fait intervenir non seulement «les vaisseaux des armateurs la plume de mes confrères / La monnaie des aveugles les mains des muets» mais aussi «les géants couverts d'algues», et «mille peuplades blanches / Dont chaque homme tenait une rose à la main» Alcools, (Cortège), p:49-50.

De plus, nous trouvons une opposition provoquée par la juxtaposition d'éléments que l'on n'attendrait pas au voisinage les uns des autres. Dans «Zone» coexistent, à propos de l'avion qui caractérise le XXème siècle dans sa modernité, «les diables dans les abîmes », les anges, «Icare Enoch Elie Apollonius de Tyane», «Les Corbeaux les faucons les hiboux», «les ibis les flamants les morabouts», «l'oiseau Roc célébré par les conteurs et les poètes», «les pihis longs et souples / Qui n'ont qu'une seule aile et qui volent par couples», «la colombe esprit immaculé / Qu'escortent l'oiseau- lyre et le paon ocellé» et enfin le phénix et les sirènes. Ici, Apollinaire invoque, dans une constellation lumineuse, «des personnages féeriques ou légendaires avec leurs gestes, leurs contours évanescents et oniriques» (12)

Abolissant systématiquement les marques de ponctuation, juxtaposant les temps, les personnes, les images, Apollinaire propose des textes visionnaires qui provoquent l'imagination du lecteur. Dans ses célèbres «Calligrammes», il fait une alliance de la peinture et de la poésie, et le texte poétique y est traité comme un dessin avec des signes du langage. La peinture cubiste a nourri sa conception de l'aventure poétique: il dissocie les 
éléments de la réalité et les combine librement de nouveau avec la nouveauté de l'écriture:

«Chez Apollinaire, non seulement les images semblent souvent avoir acquis une autonomie telle que rien, (...) mais elles s'assemblent dans une liberté qui paraît ne connaître aucun frein et se plaire parfois dans les pires disparates». (13)

L'écriture, chez Apollinaire, se caractérise par un jeu subtil entre modernité et antiquité: il ne s'agit pas de se tourner vers le passé ou vers le futur mais de suivre le mouvement du temps. «Le Calligramme» substitue donc la linéarité à la simultanéité de la forme, une création poétique visuelle qui relie la singularité du geste d'écriture à la reproduction de la page imprimée.

En fait, le lien entre le dessin du texte et son contenu est vague et il peut arriver que les mots cèdent la place à d'autres éléments tels que signes musicaux «Venu de Dieuze» ou des dessins «le faite d'un poteau télégraphique» dans «Voyage».

Dans «Voyage», le train va de gauche à droite comme l'écriture même, tandis que le son s'éloigne sur notre gauche, car il s'agit d'un aspect beaucoup plus sonore que visuel, et les éléments monosyllabiques (où, va, donc, ce train, qui, meurt, dans, les, vals, et, les, beaux, bois) répètent le bruit caractéristique du passage des roues.

Ce poème est un calligramme, dont le signifié poétique trouve son écho non seulement dans les sonorités mais aussi dans la forme graphique, il est divisé en plusieurs petits calligrammes dont l'ensemble constitue un tableau assez emblématique qui ressemble à une énigme qu'il faut déchiffrer.

C'est un récit orienté, dont le sens est donné par le terme qui se trouve à la fin de chaque figure:

«DANTE, TOMBER, PALE, PLUS»

Apollinaire y dessine un univers surréaliste et réaliste à la fois, celui de la modernité, de la technologie du XXème siècle, associant la science à l'objet poétique, science et fiction, poésie scientifique: le train, la locomotive, le télégraphe, qui voyagent comme un oiseau qui «laisse tomber ses ailes partout» Calligrammes, (Voyage), p:60.

C'est un voyage symbolique, le train avec les voyageurs incarne le trajet de la vie qui conduit les êtres humains; un voyage dont l'issue est inconnue:

«Où va donc ce train ...» Calligrammes, (Voyage), p:59.

Cette interrogation insiste sur la vie et sur la mort, sur le 
devenir de l'humanité après la mort, et elle traduit aussi l'angoisse et la désillusion. A travers ce train, la nature est incarnée par sa pérennité qui s'oppose à la brièveté de la vie humaine: «Les vals et les beaux bois»

Le paysage est fascinant, et l'été personnifié est «tendre» même s'il est «pâle» et annonce l'agonie.

Bref, «Voyage» célèbre l'aspect moderne des Années Folles: la vie, l'angoisse de la mort, le temps qui passe, la beauté de la nature qui se renouvelle au rythme des saisons.

Dans «Lettre- Océan», poème dessin ayant la forme d'un cadran, se concentre l'espace, et le poète poursuit la conquête par une suite de juxtapositions géographiques.

Dans ce poème, l'ordre visuel s'ajoute à l'ordre sonore, et l'idéogramme correspond à ce qu'il y a de synthétique chez les cubistes.

De plus, l'abondance et la variété de caractères d'imprimerie font du mot un objet pictural. Ainsi le début de «Du coton dans les oreilles» mêle majuscules et minuscules, utilise l'italique, divers caractères gras et évolue la dimension des lettres, surtout à l'intérieur du mot «Omégaphone» qui, en raison de la taille de plus en plus réduite des caractères qui le composent, semble peu à peu disparaître, comme absorbé par la page.

L'écriture se présente donc comme un acte libre, et elle témoigne du même coup de l'inépuisable polysémie du texte poétique. «Lettre- Océan» encourage la visibilité au détriment de la lisibilité, et le poème «Reconnaissance», dont les deux derniers mot sont décalés sur des lignes plus basses, semble s'achever sur un effondrement inquiétant qui oppose le mouvement contenu dans le dernier vers:

\section{«Et les canons des indolences}

Tirent mes songes vers

(Reconnaissance), p:84.

les

cieux» Calligrammes,

Le dessin du texte peut aussi démembrer un mot, voire une syllabe:

«t je fu

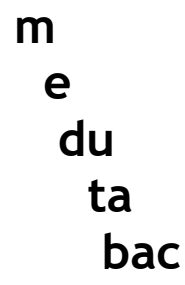


de

ZonE» Calligrammes, (Fumées), p:71.

Ces procédés de montage ou de collage se retrouvent sur le plan sonore. Apollinaire crée ainsi les poèmes- conversation, rassemblement de flux linguistique continu. Dans ces poèmes, le principe de linéarité n'existe plus, ils représentent la vie telle qu'elle est sans changement. Ce processus est basé sur des clichés de la langue, du pouvoir des images et sur une expérimentation des tours de langue (les expressions idiomatiques, les proverbes). La juxtaposition de ces propos engendre d'étranges rencontres:

«Ces crêpes étaient exquises / La fontaine coule / Robe noire comme ses ongles / C'est complètement impossible / voici monsieur» Calligrammes, (Lundi rue Christine), p:41.

Affranchi des entraves traditionnelles, Apollinaire invente sa propre musique, originale et novatrice à travers le son utilisant, outre les ressources de l'allitération et de l'assonance, celles de l'onomatopée;

«Pan pan pan

Perruque perruque

Pan pan pan

Perruque à canon» Calligrammes, (S P), p:85 et de l'interjection «Eh! Oh! Ha!» répétée six fois dans «Mutation», de l'anaphore de la forme représentative «ll y a» dans tous les vers du poème intitulé «ll y a», excepté les deux derniers, ou du mélange des langues, surtout dans «A travers l'Europe»:

«Una volta ho inteso dire Chè vuoi (...)

Mais tes cheveux sont le trolley» Calligrammes, (A travers

l'Europe), p:63.

D'autres poèmes semblent issus d'une écriture quasi

automatique, c'est pourquoi les surréalistes ont vu en Apollinaire un maître à suivre:

«Du rouge au vert tout le jaune se meurt

Quand chantent les aras dans les forêts natales

Abatis de pihis

Il y a un poème à faire sur l'oiseau qui n'a qu'une aile

Nous l'enverrons en message téléphonique

Traumatisme géant» Calligrammes, (Les fenêtres), p:25.

Bref, la démarche poétique d'Apollinaire est magistralement placée sous le signe de la liberté. L'absence de ponctuation dans «Zone» crée un poème où le lecteur a le choix d'imaginer une explication individuelle et unique. Cela montre que la réalité 
reste ambigüe , c'est pourquoi Apollinaire modifie souvent le temps du verbe. Il Passe de l'imparfait au présent «Si tu vivais dans l'ancien temps ... Vous avez honte ...». L'imparfait exprime une action répétée au passé dans un moment imprécis. Ce changement de temps présente un monde qui se transforme continuellement et par conséquent la réalité reste intouchable.

Sur le plan prosodique, si Apollinaire conserve en général la rime et la régularité métrique, c'est en raison d'une nécessité interne à sa poésie et non par soucis d'obéir à une contrainte extérieure. La répétition donne également à de nombreux poèmes un rythme qui les rapproche du cantique: «Le pont Mirabeau», par la reprise du refrain «Vienne la nuit sonne l'heure/ les jours s'en vont je demeure», et celle du premier vers «sous le pont Mirabeau coule La Seine», a l'aspect d'une litanie tragique et conjuratoire. Dans «La chanson du malaimé», la répétition d'une strophe majestueuse par son adresse et solennelle par la référence biblique qu'elle contient «Voie lactée ô sœur lumineuse / Des blancs ruisseaux de Chanaan / Et des corps blancs des amoureuses / Nageurs morts suivronsnous d'aban / Ton cours vers d'autres nébuleuses» ajoute au poème une dimension incantatoire. Alcools, (La chanson du malaimé), $\mathrm{p:30.}$

Apollinaire refuse la stabilité mélodique et préfère l'incertitude; la disposition graphique, dans «Les Colchiques» dominé par l'alexandrin, démembre le mètre: «Les vaches y paissant / Lentement s'empoisonnent», et certains vers ont plus de douze syllabes:

«Vêtus de hoquetons et jouant de l'harmonica» Alcools, (Les Colchiques), p:33.

Dans «Marie», un alexandrin unique vient soudain perturber la régularité du poème entièrement composé d'octosyllabes: «Oui je veux vous aimer mais vous aimer à peine» Alcools, (Marie), p:55.

En cela, Apollinaire s'occupe de la forme (vers libre, création lexicale) et rêve d'établir un système poétique global, sans écoles,celui du début de XXème siècle, période de renouvellement d'écriture poétique moderne, rejetant les règles étouffantes d'une poésie classique.

Dans «Zone», il n'y a pas de strophes, il y a des vers libres détachés des autres, des doublets, des tercets, des vers qui ne sont pas tous de même longueur, passant du très court au très long. 
On peut trouver des rimes suivies, des vers sans rime, et le poème peut être lu comme un poème innovant, introduisant la modernité par le biais du signifié.

Pour assurer son désir à l'écriture moderne, Apollinaire multiplie les allitérations et les rappels de sonorités qui reflètent les bruits des machines. Dans «Mai», le troisième vers «vous êtes si jolies mais la barque s'éloigne» fait écho à «le joli mai en barque» dans le premier vers. Ces rappels de sonorités se trouvent également dans «La Tzigane»:

«Nous lui dîmes adieu et puis

Tzigane), p:78.

De ce puits sortit l'Espérance» Alcools, (La

Bref, Apollinaire est un poète «créateur» des rythmes et des sons, des rimes nouvelles et il ne s'enferme Pas dans une langue dite «poétique». La variété des vers, des systèmes des rimes, des strophes est immense, et le critique Adolphe Bochot a montré que le vrai poète ne se soumet à aucun système, mais que «adoptant presque chaque jour à ses propres besoins les moyens étrangers d'expression, il invente pour lui, pendant qu'il crée chaque œuvre, une technique spéciale.» (14)

Ainsi, Apollinaire néglige la ponctuation car il a la volonté d'être un poète nouveau autant dans la forme que dans le fond:

«Le rythme même et la coupe des vers, voilà la véritable ponctuation,» (15) déclare- t- il à Henri Martineau.

Blaise Cendrars, dans «La prose du Transsibérien», présente un poème tableau. Il crée une sorte de «fantastique de réalisme» où le seul voyage exécuté est celui de l'écriture. Celle- ci obéit aux trépidations de la machine. Le vers libre est largement employé, en de longs mouvements à la strophe, qui expriment le rythme chaotique de la vie moderne.

Cendrars, comme Apollinaire, a renoncé à la ponctuation en employant une harmonie imitative puisque les vers et les mots imitent un vacarme.

Le champs lexical est très varié; celui du chemin de fer: «train, gares, route fils télégraphiques, poteaux, locomotives, roues, ferrailles, broun- roun- roun». Ce lexique introduit le visuel dans l'imagination de la scène, mais la dernière onomatopée touche surtout le site auditif «broun- roun- roun.»

De plus, le vocabulaire est accompagné d'une vision pessimiste, par l'accumulation d'adjectifs qualificatifs «gares lézardées obliques, poteaux grinçant, les roues vertigineuses , les démons déchaînés», qui montrent la destruction de Paris: 
«Paris a disparu et son énorme flambée

II n'y a plus que les cendres continues» (La prose du Transsibérien)

Valéry Larbaud fait de son poème, «Ode», une musique, une harmonie dans l'écriture, rassemblée à celle du train: «vos vibrantes voix de chanterelle», «la belle Cantatrice», «tes cent mille voix», «Ô Harmonika- Zug». Le poème devient un art poétique:

«Ah! il faut que ces bruits et ce mouvement

Entrent dans mes poèmes ... » Poésies de A- 0 Barnobooth, (Ode).

Chez Victor Segalen, l'écriture est fondée sur les sonorités, allitérations et assonances, puisqu'il n'y a pas de rimes:

«Repose- toi du son dans le silence et, du silence, daigne, revenir au son. Seul si tu peux, si tu sais être seul, déverse-toi » Stèles, (Conseils au bon voyageur)

De plus, c'est une écriture de l'intemporel: la remontée dans l'histoire jusqu'au mythe est une percée vers l'origine, la pierre est le premier lieu de l'écriture et aussi le dernier, la pierre tombale.

Pour Emile Verhaeren, son désir en faveur du vers libre montre sa passion pour la victoire de l'impressionnisme.

Verhaeren cherche des cadences nettes ponctuées par des rimes fortes. Il crée des néologismes, dont l'influence est germanique, utilisant une syntaxe chaotique avec un rythme violent né d'une association entre alexandrins et décasyllabes:

«Plus loin, un vacarme tonnant de chocs

Monte de l'ombre et s'érige par blocs, Et, tout à coup, cassant l'élan des violences, Des murs de bruit semblent tomber Et se taire, dans une marre de silence, " Les Villes tentaculaires. (Les Usines) 
En conclusion, Apollinaire présente, dans cette étude, un univers où les frontières disparaissent entre l'animé et l'inanimé, entre l'objet et la nature, soit par l'ivresse soit par la magie. Chez lui, l'ivresse est toujours poétique et le langage est magique.

Chez cet aventurier, le monde de la biologie végétale et zoologique est abordé avec des images innombrables:

«Le pré est vénéneux mais joli en automne Les vaches y paissent

Lentement s'empoisonnent» Alcools, (Les colchiques), $\mathrm{p}: 33$.

Sa tendance à la symbolisation est évidente, et tout est répété en comparaisons explicites:

«... tes yeux comme cette fleur- là

Violâtres comme leur cerne et comme cet automne Et ma vie pour tes yeux lentement s'empoisonne» Alcools, (Les colchiques), p:33.

Le colchique introduit le parallèle entre la fleur poison et la femme poison, et l'image de la fleur est féminisée dès l'indication de sa couleur, celles des cernes autour des yeux de l'aimée.

Apollinaire s'était révolté contre les formes traditionnelles, étouffantes en adoptant des méthodes nouvelles, exprimant le refus des barrières qui constituent des obstacles de l'inspiration poétique moderne.

De plus, sa poésie est donc étendue à l'espace urbain, représenté comme un lieu de bonheur grâce au lien qui existe entre chaque ville avec une poésie simple mais dont les idées sont neuves.

En un mot, la poésie moderne du XXème siècle ouvre de nouvelles visions littéraires et scientifiques après l'opposition des Anciens et des Modernes concernant le fond et la forme de l'œuvre poétique, et elle présente une vision historique assez remarquable et toujours renouvelable. 


\section{Les notes}

1- Décaudin (Michel): «Le changement de front d'Apollinaire». in Revue des sciences humaines. ${ }^{\circ} 60$ Octobre- Décembre, 1950.

2- Delesalle (S): «La modernité dans le langage d'apollinaire». in Europe. numéro spécial, Novembre- Décembre, n451-452, 1966.

3- Bachelard (Gaston): Psychanalyse du feu. Gallimard, 1938. p:167.

4- Renaud (Philippe): Lecture d'Apollinaire. Editions l'Age d'Homme, La cité Lausanne, 1969. p:118.

5- Ibid. p:161.

6- Cendrars (Blaise): GEuvres complètes. «L'Homme foudroyé». Editions Denzoël, tome V, 1913. p:49.

7- Bachelard (Gaston): L'air et les songes. José Corti, 1943. p:12.

8- Durry (Marie- Jeanne): Guillaume Apollinaire. Alcools. Tome II, Sèdes, 1979. p:199.

9- Tassari (Roberto): «Le futurisme et la machine». in Europe. Mars, 1975.

10- Chadourne (Jacqueline): Blaise Cendrars poète du cosmos. Editions Seghers, Paris, 1973. P:199.

11- Apollinaire (Guillaume): L'Esprit nouveau et les poètes. Paris, Haumont, 1946. p:17.

12- Hubert (Renée Riese): «Le langage des merveilles et des surprises». in Lettres Modernes. Ménard, 1973.

13- Décaudin (Michel): Le dossier d'Alcools. Editions Minard, 1960. 
14- Décaudin (Michel): Alcools de Guillaume Apollinaire. Gallimard, 1989. p:69.

15- Oster (Daniel): Guillaume Apollinaire. Seghers, Paris, 2001. p:107. 


\section{BIBLIOGRAPHIE}

\section{I- Corpus:}

1- Apollinaire (Guillaume): Alcools. Editions Gallimard, 1920.

2- Apollinaire (Guillaume): Calligrammes. Editions Gallimard, 1925.

II- Guvres de poète:

1- Apollinaire (Guillaume): L'Esprit nouveau et les poètes. Paris, Hautmont, 1946.

\section{III- Ouvrages de critique:}

A- Ouvrages de critique consacrés au poète:

1- Daudin (Claire): Connaissance d'une œuvre, Guillaume Apollinaire. Bréal, 1998.

2- Décaudin (Michel): Alcools de Guillaume Apollinaire. Gallimard, 1989.

3- Décaudin (Michel): Le dossier d'Alcools. Editions Minard, 1960.

4- Durry (Marie- Jeanne): Guillaume Apollinaire. Alcools. tome II, Sèdes, 1979.

5- Oster (Daniel): Guillaume Apollinaire. Seghers, Paris, 2001.

6- Renaud (Philippe): Lecture d'Apollinaire. Editions L'Age d'Homme, La Cité Lausanne, 1969.

$B$ - Ouvrages généraux:

1- Bachelard (Gaston): Psychanalyse du feu. Gallimard, 1938.

2- Bachelard (Gaston): L'air et les songes. José Carté, 1943.

3- Bezbakh (Pierre): Histoire de la France contemporaine, de 1914 à nos jours. Bordes, Paris, 1990.

4- Brée (Germaine), Marot- Sir (Edouard): Littérature française, Du surréalisme à l'Empire de la critique. Arthaud, Paris, 1984.

5- Cendrars (Miriam): Blaise Cendrars. La vie, le verbe, l'écriture. Paris, Denoël, 2006. 
6- Chadourne (Jacqueline): Blaise Cendrars poète de cosmos. Editions Seghers, Paris, 1973.

7- Chaudier (Stéphane), Lioure (Françoise): Les langages de Larbaud. Presses universitaires Blaise Pascal, Collection «Littératures», 2006.

8- Guyon (Laurence): Cendrars en énigme. Modèles religieux, écritures poétiques. Paris, Champion, Collection «Cahiers Blaise Cendrars», $n^{\circ}$ 9, 2007.

\section{IV- Les périodiques:}

1- Décaudin (Michel): «Le changement de front d'Apollinaire». in Revue des sciences humaines. $\mathrm{n}^{\circ}$ 60, Octobre- Décembre, 1950.

2- Dos Passos (John): «Homère du transsibérien». in Orient Express. 1987.

3- Hubert (Renée Riese): «Le langage des merveilles et les surprises». in Letters Modernes. Minard, 1973.

4- Marten (Van Buuren): «Le dilettantisme, style de vie». in Poétique. Paris, France, $\mathrm{n}^{\circ}$ 137, 2004.

5- Perse (Saint- John): «Une philosophie au miroir de la poétique». in La philosophie du XXe siècle et le défi poétique, $\mathrm{n}^{\circ} 7,2004$.

6- Tassari (Roberto): «Le futurisme et la machine». in Europe, Mars, 1975.

7- Sidoti (Antoine): «Genèse et dossier d'une polémique la prose du Transsibérien et de la petite Jehanne de France, Blaise Cendrars. Sonia Delaunay». Paris, Minard, in Archives des Lettres Modernes. Novembre- Décembre, $n^{\circ} 224,1987$.

\section{V- Les Dictionnaires:}

1- Dictionnaire des œuvres littéraires de langue française. Bordas, Paris, 1994.

2- Petit Robert, Dictionnaire de la langue française. Montréal, Canada, 1990.

\section{VI- Sites internets:}

1- http://www.france web.fr/poésie/transib.htm.

2- http://fr.wikipedia.org/wiki/guillaume Apollinaire

3- http://fr.wikipedia.org/Modernit\% C3\% A9 


\section{TABLE DES MATIERES}

I- Introduction

p: 2

II- L'eau et le feu

p: 3

III- La machine et la poésie

p: 11

IV- La ville

p: 15

V-L'écriture apollinairienne

p: 20

VI- Conclusion

p: 27

VII- Les notes

p: 28

VIII- Bibliographie

P: 30

IX-Table des matières

p: 32 